\section{The Infrared Association Band of a Heavy Alcohol}

By comparing the infrared absorption spectra of different alcohols in the region of the fundamental $\mathrm{O}-\mathrm{H}$ vibration in gaseous and liquid state it is possible to draw some conclusions about the association. Several workers have studied these association bands, most in the region of the harmonics. Errera and Mollet ${ }^{1}$ found for solutions of $\mathrm{C}_{2} \mathrm{H}_{6} \mathrm{OH}$ in $\mathrm{CCl}_{4}$ two bands, one at $3640 \mathrm{~cm}^{-1}(2,73 \mu)$ and a broad band at about $3350 \mathrm{~cm}^{-1}(3 \mu)$. The first is ascribed to the monomolecules and the second to intermolecular $\mathrm{O}-\mathrm{H}$ vibrations. The temperature effect is in accord with this supposition, for increasing the temperature decreases the intensity of the broad intermolecular band and at the same time increases that of the monomolecular band. As was expected the vapor state shows a sharp monomolecular band. The methyl band at $3.4 \mu$ is not shifted.

We were able to find an analogous effect for water by comparing the absorption in the gaseous, liquid and solid states, and in solutions of water in $\mathrm{CCl}_{4}, \mathrm{CS}_{2}$ or dioxane. ${ }^{2}$ Many suggestions have been given as to the interpretation of these association bands. ${ }^{3}$ It is however not yet possible to draw any definite conclusions about the character of these complexes from infrared data.

The isotope effect has proved to be very helpful in the explanation of differents bands. In studying the finestructure of one of the heavy alcohols we found the same effect as described above. The methylband (in this case $\mathrm{CH}_{2} \mathrm{D}$ of the molecule $\mathrm{CH}_{2} \mathrm{DOD}$ ) remains in the same place for the gaseous and the liquid state. The monomolecular band at $2721 \mathrm{~cm}^{-1}$ disappears in the liquid state (only a small inflection is to be seen) and a broad association band is found at about $2500 \mathrm{~cm}^{-1}$. These data are obtained with a prism grating instrument. The transmission curve is composed of three parts due to the changing of the fore-prism. The methyl band in the spectrum of the vapor state was measured by lowering the pressure of the alcohol vapor.

The $\mathrm{O}-\mathrm{D}$ band at $2721 \mathrm{~cm}^{-1}$ proved to have the features of a parallel band and has a fine structure with a sharp zero branch. These results will be published later.

Bartholomé and Sachsse measured the spectrum of $\mathrm{CH}_{3} \mathrm{OH}$ and $\mathrm{CH}_{3} \mathrm{OD}$ only with prisms of $\mathrm{CaF}_{2}$ and $\mathrm{NaCl}$. They found a methyl band at $3000 \mathrm{~cm}^{-1}$ and the $\mathrm{O}-\mathrm{H}$ and $\mathrm{O}-\mathrm{D}$ stretching vibrations respectively at 3400 and

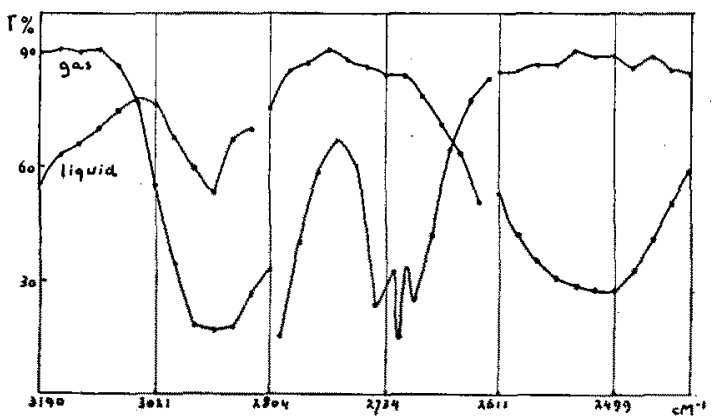

FIG. 1. The transmission curve of $\mathrm{CH}_{2} \mathrm{DOD}$ in the gaseous and liquid state.
$2420 \mathrm{~cm}^{-1}$. These data do not agree with the results of Errera and Mollet, nor with our measurements. We must suppose that in their cell a small amount of alcohol is condensed on the windows. This is very possible for a very thin layer of alcohol (only a few drops between two polished plates of $\mathrm{KBr}$ of $5 \mathrm{~cm}^{2}$ surface) gave us the association band as Fig. 1 shows. The $\mathrm{O}-\mathrm{D}$ band moreover is rather sharp and it was studied by these authors with the fluorite prism which has a very small dispersion in this region.

Also, the Raman spectra have been studied recently by Halford, Anderson and Kissin. ${ }^{5}$ They find the methyl bands at $2890 \mathrm{~cm}^{-1}$ and $2987 \mathrm{~cm}^{-1}$ (we also resolved the methyl band at $2900 \mathrm{~cm}^{-1}$ ) and ascribe the broad band at $3400 \mathrm{~cm}^{-1}$ to the $\mathrm{O}-\mathrm{H}$ stretching motion and the smaller band at $2500 \mathrm{~cm}^{-1}$ to the $\mathrm{O}-\mathrm{D}$ stretching motion. Also in this case it is our opinion that these bands are due to association since their observations were carried out in the liquid state.

We thank Professor L. C. Anderson of the department of chemistry for permitting us to use a sample of the heavy alcohol and Professor E. F. Barker for his kind interest.

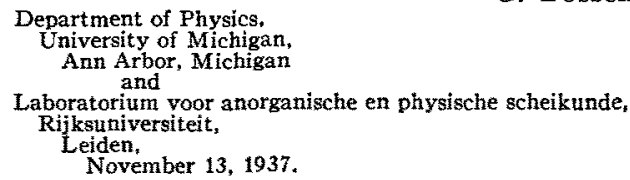

' J. Errera and P. Mollet, Nature 138, 882 (1936); Trans. Faraday Soc. 33, 122 (1937); Comptes rendus 204, 259 (1937).

${ }^{2} \mathrm{G}$. Bosschieter and J. Errera. J. de physique 8, 229 (1937) and Comptes rendus 204, 1719 (1937); 205, 560 (1937).

3 R. M. Badger and S. H. Bauer, J. Chem. Phys. 5, 839 (1937) - E. Bartholomé and H. Sachsse, Zeits. f. physik. Chemie B30, 40 (1935).

J. O. Halford, L. C. Anderson, G. H. Kissin, J. Chem. Phys. 5, 927 (1937)

\section{The Raman Spectra of $\mathrm{CH}_{3} \mathrm{CF}_{3}$ and $\mathrm{CCl}_{2} \mathrm{CF}_{2}{ }^{*}$}

Because of the interest attached to ethane and ethanelike molecules in connection with the question of free rotation of the $\mathrm{CX}_{3}$ groups with respect to each other, it was considered that the Raman spectrum of $\mathrm{CH}_{3} \mathrm{CF}_{3}$ might yield additional information on this point. Inasmuch as the spectrum of the liquid can be conveniently photographed only at low temperatures (b.p. about $-40^{\circ}$ ), the determination of the polarization of the scattered light would be experimentally very difficult and was not attempted. The fact that the frequencies associated with the $\mathrm{CF}_{3}$ group will be considerably different in magnitude from those of the $\mathrm{CH}_{3}$ group may, in the analysis, compensate for the lack of data on polarization. Only the results of the experiments are presented here; the assignment of the frequencies will be given when completed. The Raman spectrum of the ethylene-like molecule $\mathrm{CCl}_{2}=\mathrm{CF}_{2}$ was photographed at room temperature with the substance in the liquid state, and the observed shifts are presented here.

The compounds were kindly furnished us by Dr. A. $L$. Henne in a pure or nearly pure state to begin with, and they were further fractionally distilled before placing them in the Pyrex or quartz Raman tubes. Spectra of $\mathrm{CH}_{3} \mathrm{CF}_{3}$ gas and liquid, and $\mathrm{CF}_{2} \mathrm{CCl}_{2}$ liquid were photographed, 
TABLE I. The Raman spectrum of $\mathrm{CH}_{3} \mathrm{CF}_{3}$ liquid at $-75^{\circ}$.

\begin{tabular}{clc}
\hline \hline $\begin{array}{c}\text { Frequency } \\
\text { in } \mathrm{cm}^{-1}\end{array}$ & Character & $\begin{array}{c}\text { Number of Exciting } \\
\text { Lines Scattered from }\end{array}$ \\
\hline $358(2)$ & & 2 \\
$541(2)$ & Sharp & 3 \\
$603(2)$ & Sharp & 2 \\
$829(5)$ & Sharp & 5 \\
$968(3)$ & & 3 \\
$1279(1)$ & & 1 \\
$1450(4)$ & Diffuse & 2 \\
$2792(2)$ & & 1 \\
$2825(1)$ & & 2 \\
$2885(1)$ & Diffuse & 1 \\
$2974(5)$ & & 8 \\
$3040(4)$ & Diffuse & 5 \\
\hline
\end{tabular}

TABLE II. The Raman spectrum of $C F_{2}=C C i_{2}$ liquid at room temperature.

\begin{tabular}{clc}
\hline \hline $\begin{array}{c}\text { Frequency } \\
\text { in } \mathrm{cm}^{-1}\end{array}$ & Character & $\begin{array}{c}\text { Number of Exciting } \\
\text { Lines Scattered from }\end{array}$ \\
\hline $255(6)$ & & 7 \\
$435(7)$ & Sharp & 4 \\
$454(1)$ & & 2 \\
$562(6)$ & Sharp & 5 \\
$622(4)$ & Diffuse & 2 \\
$648(2)$ & Diffuse & 1 \\
$883\left(\frac{1}{2}\right)$ & Diffuse & 2 \\
$1027(2)$ & & 2 \\
$1123(1)$ & Diffuse & 2 \\
$1735(6)$ & 2 \\
$1976\left(\frac{1}{2}\right)$ & \\
\hline
\end{tabular}

the exposure times varying from one to twenty hours in the case of the liquids. A quinine hydrochloride solution filter between the mercury arc and the tube was used in some exposures on both compounds to determine which of the observed lines were excited by $4358 \mathrm{~A}$ and which by 4078 or $4047 \mathrm{~A}$. This test or an equivalent one is necessary for those cases in which the scattered lines from two or more exciting lines overlap. In Table I the numbers in parentheses adjoining the frequency values indicate estimated relative intensities.

Since $\mathrm{CH}_{3} \mathrm{CF}_{3}$ gas absorbs fairly strongly in the ultraviolet, the results of experiments on it are not presented separately, and they were of use only in confirming the 3040,2974 and $829 \mathrm{~cm}^{-1}$ lines. The absorption resulted in the photochemical decomposition of the gas. The substance in the liquid form is colorless and showed no absorption in the visible region.

The selection rules ${ }^{1}$ for $\mathrm{CH}_{3} \mathrm{CF}_{3}$, assuming a symmetry of $C_{3 v}$, permit a total of 11 fundamental frequencies in the Raman spectrum, of which 6 would have a polarization $\rho=\frac{3}{4}$. Twelve lines in all were observed, of which the one with a frequency of $2885 \mathrm{~cm}^{-1}$ is the only doubtful one. For purposes of comparison it is of interest to set down here the vibrational frequencies obtained for $\mathrm{CF}_{4}$ in the Raman spectrum ${ }^{2}$ and infrared; ${ }^{3} \nu_{1}(1), 904 ; \nu_{2}(2), 437$; $\nu_{3}(3), 1252 ; \nu_{4}(3), 635 \mathrm{~cm}^{-1}$. The selection rules for $\mathrm{CF}_{2}$ $=\mathrm{CCl}_{2}$ permit 12 fundamental frequencies to appear in the Raman spectrum ( 7 with $\rho=\frac{3}{4}$ ) if the symmetry is assumed to be $C_{2 v}$, while only 11 were observed. Moreover some of the weaker lines may be combinations of overtones. JoHN B. Hatcher

\section{Gates Chemical Laboratory, \\ California Institute of Technology, \\ Pasadena, California}

* Contribution from the Gates and Crellin Laboratories of Chemistry, California Institute of Technology, No. 630 .

1 The selection rules for the $\mathrm{CH}_{3} \mathrm{CF}_{3}$ type molecule were determined by Dr, Fred Stitt.

2 Yost, Lassettre and Gross, J. Chem. Phys, 4, 325 (1936)

3 C. R. Bailey, Hale and Thompson. J. Chem. Phys. 5, 274 (1937) Eucken and Bertram, Zeits. f. physik. Chemie B27, 79 (1934).

\section{The Separation of Mixtures by Centrifuging}

The separation of a mixture into its pure constituents by the usual methods such as fractional distillation, fractional crystallization, etc., sometimes is a very slow and tedious process. This is especially true in the case of many constant boiling point mixtures. Therefore, it might be of interest to mention some experiments in which our vacuum type tubular centrifuge was used to separate a mixture of substances of different molecular weights while in the vapor state.

Briefly, the centrifuge consists of a vacuum tight alloy steel tube $2.6 \mathrm{~cm}$ (effective) internal diameter and 12.5 $\mathrm{cm}$ long, which spins inside a vacuum chamber.1 The vapor entered the spinning tube at the top through a stainless steel hypodermic needle tube (gauge 15), and the lighter and heavier fractions were collected at the bottom through two coaxial stainless steel hypodermic needle tubes (gauges 15 and 12), communicating with the axis of rotation and the periphery of the spinning tube, respectively. The inlet to the spinning tube was sealed (by vacuum tight oil glands) to a flask containing a mixture (constant boiling point) of carbon tetrachloride and methyl alcohol, and the outlets which collected the lighter and heavier fractions were sealed, also by vacuum tight oil glands, each to a separate trap immersed in dry ice. The system was evacuated and the vapor passed through the centrifuge at the rate of approximately a cubic centimeter of liquid per minute. In each experiment precaution was taken to make sure that practically all of the mixture placed in the flask passed through the centrifuge and was collected in the dry-ice traps; also, that approximately equal light and heavy fractions were collected. Care was taken to insure that each fraction contained only the carbon tetrachloride-methyl alcohol mixture and hence was free of possible impurities such as vacuum pump oil, etc. With the tube or centrifuge spinning 1800 r.p.s. the density of the heavier fraction in the liquid state was roughly 4.7 percent heavier than that of the lighter fraction in the liquid state at the same temperature.

According to the well-known theory for the centrifuging of gases $^{2}$ when equilibrium is established

$$
K_{p} / K_{p}=e^{\left(M_{1}-M M_{2}\right) w^{2} r^{2} / 2 R T}
$$

where $M_{1}$ and $M_{2}$ are the molecular weights of the two gases, respectively, $T$ the absolute temperature, $R$ the gas constant, $r$ the radius of the centrifuge, $w$ the angular velocity in radians per sec., $K_{p}$ the ratio of the quantities 